\section{Prediction of psychosis: setting the stage}

\author{
ALISON R. YUNG and PATRICK D. McGORRY
}

Summary Treating psychotic disorders in their earliest stages has become a key focus for research and clinical care. This paper reviews evidence of the capacity to identify those at increased risk for psychotic disorder and to intervene in the identified, high-risk individuals to ameliorate the course of disorder. Issues involved in preventive oriented clinical care are addressed, such as risk/benefit considerations, ethical and safety issues and the value of stage-specific interventions. Clinical predictors identified in recent research, promising intervention trials and proposed clinical practice guidelines are described. An approach based on active engagement, support and monitoring, yet with a conservative approach to medication use is advocated at present. Potential neurobiological processes have been studied and reinforce the sense that this is a critical phase for active treatment, and may prove helpful in understanding the process of transition across stages of illness. More research is required in prediction, neurobiology and treatment.

\section{Declaration of interest None.}

\footnotetext{
'The best hope now for the prevention of schizophrenia lies with indicated preventive interventions targeted at individuals manifesting precursor signs and symptoms who have not yet met full criteria for diagnosis. The identification of individuals at this early stage, coupled with the introduction of pharmacological and psychosocial interventions, may prevent the development of the full-blown disorder' (Mrazek \& Haggerty, 1994 p. I54).
}

Such sentiment underlines the aim of identifying people in the prodromal phase preceding a first psychotic episode. If an impending psychotic disorder can be recognised, then the possibility of prevention, or at least delay of or lessening the severity and mode of onset, arises. For over a decade our team has been researching this field. A first step was to characterise the typical features of the prepsychotic phase and their course and natural history, particularly with regard to the timing and onset of full-blown psychosis. Detailed studies of psychotic prodromes revealed that neurotic and mood-related type symptoms were common, including depressed mood, anxiety, sleep disturbance and irritability (Yung \& McGorry, 1996). Indeed, Hafner et al (2005) found that the prodrome of schizophrenia was indistinguishable from that of major depression in its early stages. Negative and 'basic' symptoms have also been described in prodromes. Basic symptoms refer to subjectively experienced abnormalities in the realms of cognition, attention, perception and movement and are thought by many to be important precursors of schizophrenia (Klosterkotter et al, 1997). Finally, attenuated (or sub-threshold) psychotic symptoms are also features of the psychotic prodrome, typically occurring late, just prior to the development of fullblown psychotic symptoms (Yung \& McGorry, 1996). See Appendix for a summary of some of the commonly occurring features of the prodrome.

\section{UTILITY OF A CLINICAL STAGING MODEL}

Individuals experiencing a prodrome are conceptualised as being in the early stages of a psychotic disorder such as schizophrenia. The prodrome concept is therefore an example of the application of a clinical staging model to psychiatry (McGorry et $a l, 2006)$. Clinical staging has long been used in general medicine; however, it has been neglected in psychiatry (Fava \& Kellner, 1993). The model is particularly well established in the treatment of malignancies, where quality of life and survival are thought to rely on earliest possible delivery of effective interventions. We argue that at the heart of the concept is the differentiation of initial and milder clinical phenomena from those that accompany illness extension, progression and chronicity (McGorry et al, 2006).

Although a clinical staging model links treatment selection and prediction, its role in treatment selection is more crucial, particularly since early successful treatment may change the prognosis and potentially prevent progression to subsequent stages. The fundamental assumptions of clinical staging are twofold: that patients in the early stages of an illness have a better response to treatment and a better prognosis than those in later stages, and that the treatments offered in the early stages should be more benign as well as more effective. Table 1 shows a framework for a clinical staging model in psychotic disorders.

However, the prodrome concept, and its implications for early diagnosis and treatment, runs into problems due to the wide variability between individuals and the lack of specificity of many of its features. That is, many prodromal symptoms and signs are non-specific and could be the result of a number of conditions, such as major depression, substance misuse and physical illness, as well as a psychotic prodrome. Even attenuated or isolated psychotic symptoms may not necessarily progress to a frank psychotic disorder, as these are now known to be quite common in the general population (Tien, 1991; van Os et al, 2001). This false-positive problem has also been addressed in the staging models for cancer and other medical settings. Clinical experience and this recent research have shown that there are many people with something resembling the clinical phenotype of psychosis who apparently do not have a need for care (van Os et al, 2001) and most people who do develop a sustained psychotic disorder experience a significant period of sub-threshold symptoms, distress and serious functional decline long before they become frankly psychotic and ultimately access treatment (Yung $\&$ McGorry, 1996). However, while we may wish to 'protect' one group from intervention or at least not seek them out, we must try to find ways to offer it to another. We therefore need to decide who needs care, how early and where it should be offered, and what should be the range and sequence of interventions. Hence the challenge is to identify individuals with a high likelihood 
Table I Clinical staging model framework for psychotic disorders (modified from McGorry et al, 2006)

\begin{tabular}{llll}
\hline $\begin{array}{l}\text { Clinical } \\
\text { stage }\end{array}$ & Definition & Target populations for recruitment & Potential interventions \\
\hline 0 & Increased risk of psychotic disorder & First degree teenage relatives of & Improved mental health literacy \\
& No symptoms currently & probands & Family education, drug education \\
& & Brief cognitive skills training
\end{tabular}

Ia

Mild or non-specific symptoms, including neurocognitive deficits Mild functional change or decline

Screening of teenage populations Referral by primary care physicians Referral by school counsellors

Ultra-high risk: moderate but subthreshold symptoms, with moderate neurocognitive changes and functional decline to caseness
Referral by educational agencies, primary care physicians, emergency departments, welfare agencies
Formal mental health literacy

Family psychoeducation, formal cognitive-behavioural therapy Active substance misuse reduction Family psychoeducation, formal cognitive-behavioural therapy Active substance misuse reduction cognitive-behavioural therapy Active substance misuse reduction Atypical antipsychotic agents for episode Vocational rehabilitation As for stage 2 with additional emphasis on medical and psychosocial strategies to achieve full remission

As for stage 3a with additional Continue with markers of illness emphasis on relapse prevention and state, trait and progression 'early warning signs' strategies

Continue with markers of illness state, trait and progression

Could be linked or fast-tracked to stage 4 disorder which stabilises with treat- services ment at a level of GAF, residual symptoms, or neurocognition below the best level achieved following remission from first episode Multiple relapses, provided worsen- Specialist care services ing in clinical extent and impact of illness is objectively present

4 Severe, persistent or unremitting Specialised care services illness as judged on symptoms, neurocogntion and disability criteria Note: could fast track to this stage at first presentation through specific clinical and functional criteria (from stage 2) or alternatively by failure to respond to treatment (from stage $3 a$ )
Indicative biological and endophenomarkers

\section{Niacin sensitivity, folate status, magnetic resonance imaging and magnetic resonance spectroscopy changes, hypothalamic-pituitary- adrenal axis dysregulation Continue with markers of illness state, trait and progression \\ Trait marker candidates and eye movements, $\mathrm{P} 50$, niacin sensitivity, mismatch negativity, Trait and state candidates where feasible according to sample size}


and young adulthood (Hafner et al, 1994). In addition, clinical risk factors, such as functional decline and putatively prodromal symptoms, particularly those hypothesised to occur immediately before the onset of frank psychosis, such as attenuated and isolated psychotic symptoms, are included. Individuals meeting these combinations of risk factors are called 'ultra-high risk' (UHR), the term 'ultra' being added to distinguish these individuals from subjects in traditional genetic high-risk studies. The ultra high risk approach attempts to identify individuals at risk for a brief period (1-2 years); that is, they are considered to be potentially in a state of incipient psychotic disorder or possibly 'prodromal' (or clinical stage $1 \mathrm{~b}$, see Table 1 ). The current ultra-high risk criteria require that a young person be aged between 14 and 29 years, is referred for help to a clinical service and meets criteria for one or more of the following groups:

(a) attenuated psychotic symptoms group: have experienced sub-threshold, attenuated positive psychotic symptoms during the past year

(b) brief limited intermittent psychotic symptoms group (BLIPS): have experienced episodes of frank psychotic symptoms that have not lasted longer than a week and have been spontaneously abated

(c) trait and state risk factor group: have schizotypal personality disorder or a first-degree relative with a psychotic disorder and have experienced a significant decrease in functioning during the previous year.

These criteria are described in more detail elsewhere (Yung et al, 2003, 2004).

Criteria have also been developed to define the onset of frank psychotic disorder. These are not identical to DSM-IV criteria, but are designed to define the minimal point at which antipsychotic treatment is indicated. This definition is arbitrary, and is defined by the persistence of frank psychotic symptoms for over 1 week.

We tested the ability of the ultra high risk criteria to identify individuals likely to develop a psychotic disorder within a brief follow-up period in a specialised service, the Personal Assessment and Crisis Evaluation (PACE) Clinic (Yung et al, 1996). As hypothesised, meeting the ultra high risk criteria was associated with a high rate of onset of psychotic disorder, with over $40 \%$ making the transition within 12 months in an initial study (Yung et al, 2003). Although the rate has seemingly reduced in a subsequent investigation, meeting the criteria stills significantly predicts transition compared to not meeting criteria (Yung et al, 2006).The ultra-high risk criteria have been adopted and adapted by a number of other settings around the world, with 12month transition rates varying between 10 and $50 \%$ (for a summary see Haroun et al, 2006 and Olsen \& Rosenbaum, 2006).

\section{Defining risk status by basic symptoms}

The Bonn group in Germany used a different approach to defining those they considered at high risk of schizophrenia (Klosterkotter et al, 1997, 2001). They studied the predictive capacity of the basic symptoms in a cohort of non-psychotic patients attending a tertiary referral psychiatric setting and who were suspected as being 'susceptible to schizophrenia' on the basis of their psychopathology. Participants were followed up on average eight years after initial assessment, and during this period over $50 \%$ of them had developed schizophrenia. Certain basic symptoms (disturbances of receptive speech, blocking of thoughts, visual perceptual disturbances, olfactory, gustatory and other sensory disturbances) were found significantly more often in the group which developed schizophrenia compared to the group which did not, suggesting that these symptoms may be predictors of schizophrenia. From this study, the authors developed a checklist of nine symptoms suggestive of a schizophrenia prodrome: inability to divide attention, thought interference, thought pressure, thought blockages, disturbance of receptive speech, disturbance of expressive speech, disturbances of abstract thinking, unstable ideas of reference and captivation of attention by details of the visual field (Klosterkotter et al, 1997). High-risk criteria were then developed requiring the presence of at least two of these symptoms. The predictive validity of these criteria is currently being examined in a multi-site European study (Klosterkotter $e t$ $a l, 2005 b)$.

\section{Recognition and prevention criteria}

Another approach to identifying high-risk individuals has been undertaken at the Hillside Recognition and Prevention (Hillside-RAP) programme in New York (Cornblatt et al, 2002). Investigators at this site, who bring their expertise from genetic high-risk studies to the area of prepsychotic research, have schizophrenia as their target syndrome, rather than psychosis more broadly. Accordingly some of their intake and outcome criteria have been modified from the PACE criteria. The RAP Clinic has two categories of 'clinical high-risk' (CHR) patients. The three intake groups in the RAP Clinic are

(a) clinical high-risk (negative) group, which includes young people displaying attenuated negative symptoms such as social isolation, avolition, and deterioration of role (academic) functioning,

(b) clinical high-risk (positive) group, which consists of adolescents with attenuated positive psychotic symptoms, and

(c) schizophrenia-like psychosis' (SLP) group (those with psychotic symptoms but not meeting criteria for schizophrenia).

The above three approaches all attempt to identify individuals in what we would term clinical stage $1 \mathrm{~b}$ : that is, the populations are symptomatic and help-seeking, and are suspected of being in the prodrome of psychosis. However, none of these sets of criteria is perfect, and a greater proportion of people who meet the criteria remain without psychosis than progress to psychosis. Further refinement is needed and investigation of those within these samples who are at particularly high risk is required. The following section briefly summarises data about possible predictors within these stage $1 \mathrm{~b}$ cohorts.

\section{PREDICTING PSYCHOSIS IN HIGH-RISK GROUPS}

Within prodromal or ultra-high risk services, numerous clinical and psychopathological variables have now been shown to predict the onset of psychosis, including factors that are not directly related to the ultra-high risk inclusion criteria.

\section{Schizotypal personality features}

Schizotypal features within some high-risk samples have been found to predict the onset of schizophrenia. In one study, odd beliefs and magical thinking at baseline were salient predictors of psychosis at followup (Mason et al, 2004). However, it is not clear in this research whether schizotypal personality truly preceded the onset of the psychotic disorder, or if in retrospect these 
were prodromal manifestations of the illness. In another study, (the Edinburgh High Risk study) participants who developed schizophrenia over the follow-up period had initially higher levels of social withdrawal, social anxiety introversion and odd behaviour (Johnstone et al, 2005). This is of note as these participants were not presenting to a clinical service, but rather were identified through relatives affected by psychotic disorders. However, the concept and measurement of schizotypy requires further examination, as items consist of a mixture of positive psychotic-like experiences, magical thinking, and negative symptoms, which make these findings difficult to interpret.

\section{Positive psychotic phenomena}

Attenuated or sub-threshold psychotic symptoms form the basis of most ultra-high risk and prodromal intake criteria in centres throughout the world. Several individual psychotic-like symptoms have also been found to be predictive of transition to full-blown psychotic disorder, including unstable ideas of reference and visual and auditory perceptual disturbances (Klosterkotter et al, 2001; Mason et al, 2004). Elevated scores on measures of unusual thought content, suspiciousness, perceptual disturbance and conceptual disorganisation (for example the Brief Psychiatric Rating Scale (BPRS) positive symptoms scale) have similarly been found to be significant predictors of psychosis at 12-month follow-up (Yung et al, 2003; Haroun et al, 2006). This is not surprising as the onset of psychosis is not a qualitative change from the sub-threshold state, instead requiring the worsening and persistence of these symptoms rather than any acquisition of new phenomena or other changes. However, it may be that some sub-threshold psychotic symptoms or psychotic-like experiences are more likely to progress than others, and some may be more likely to be associated with functional decline and poor outcome (Yung et al, 2006).

\section{Negative symptoms}

High levels of baseline negative symptoms have been observed in several ultra-high risk or prodromal samples (Lencz et al, 2004; Yung et al, 2003, 2004), despite not being part of the standard inclusion criteria. Symptoms which have been found to be predictive of psychosis include impaired concentration and attention, subjectively abnormal emotional experiences, impaired energy and impaired tolerance to stress (Yung et al, 2005), marked impairment in role functioning, anhedonia and asociality (Mason et al, 2004), blunted affect (Mason et al, 2004; Yung et al, 2005) and social withdrawal (Johnstone et al, 2005; Haroun et al, 2006). These are of interest as they may reflect an underlying abnormality, particularly in schizophrenia and may be associated with cognitive changes.

\section{Basic symptoms}

Basic symptoms overlap with negative symptoms and are regarded by some as the subjective manifestation of negative symptoms (Klosterkotter et al, 1997). Thus many of the subjectively defined negative symptoms above could also be seen as basic symptoms. The Cologne Early Recognition (CER) project examined the predictive validity of clusters of basic symptoms in a sample of patients referred to five German psychiatric out-patient services for diagnostic clarification, on the basis of suspected incipient schizophrenia. The cluster of thought, language, perception and motor disturbances was found to have good predictive ability in a receiver operator analysis (ROC) analysis (Klosterkotter et al, 2001), such that having any five of the 35 items pertaining to these symptoms at baseline predicted onset of schizophrenia after a mean follow up of 9.6 years (specificity $=0.84$, sensitivity $=0.56$, positive predictive value $=0.77$ and negative predictive value $=0.66$ ). Therefore, $77 \%$ of those with five or more symptoms of thought, language, perception or motor disturbances at baseline went on to develop schizophrenia. However, that participants in this study were all suspected of having emerging schizophrenia limits the generalisability of the results to community samples or even cohorts of help-seekers who are not suspected of being prodromal. Again these basic symptoms are of interest as they may reflect a fundamental brain abnormality in schizophrenia.

\section{Depression, anxiety and distress}

Depression has been found to be a significant predictor of psychosis in ultra-high risk groups (Yung et al, 2003; Johnstone et al, 2005). Indeed in community samples, individuals who experience distress or depression related to their psychotic-like experiences are more likely to seek help compared to their counterparts without such accompanying symptoms (Hanssen $e t$ al, 2005; Krabbendam et al, 2005). Distress and depression in relation to psychotic experiences have also been found to be associated with poor psychosocial functioning (Yung et al, 2006). These findings have prompted researchers to propose that distress, anxiety, depression and other forms of affective disturbance may play a major role in determining whether young people with psychotic symptoms progress to develop psychotic disorder (Escher et al, 2002; Freeman \& Garety, 2003; Broome et al, 2005).

\section{Poor functioning}

Poor functioning at intake predicted onset of psychosis in several separate ultra-high risk cohorts (Yung et al, 2003, 2006; Mason et al, 2004, 2006). This may indicate that a deterioration process, and actual onset of psychotic disorder, has already begun in those ultra-high risk subjects with poor functioning. However, the process may be more dynamic than this; young people with more functioning may be less able to cope with psychotic experiences, more susceptible to depression and distress, more likely to use substances and have fewer social supports than their better functioning counterparts. Hence a vicious cycle may develop in which psychotic experiences worsen in response to these factors until eventually the threshold is crossed and disorder is deemed to have begun.

\section{Substance use}

Prospective studies suggest that substance use can contribute to the onset of schizophrenia in individuals, irrespective of an identified risk for illness (e.g. Thornicroft, 1990). The evidence to date is most consistent for cannabis use (Zammit, 2002). An association between cannabis use and increased likelihood of psychotic relapse has also been described (Linszen et al, 1994), as has an association between cannabis use and increased levels of psychotic experiences in non-clinical general population surveys (Johns et al, 2004).

The role of cannabis in the onset of psychosis in ultra-high risk or prodromal groups is therefore important to investigate. The longitudinal studies in this area report contrasting findings. For example, a history of substance misuse was present in significantly more subjects who developed psychosis in the Haroun et al (2006) sample, 
although, contrary to expectations, neither cannabis use nor dependence in the year prior to contact with services was associated with a higher risk of developing psychosis over the following year in the PACE cohort (Phillips et al, 2002a). The PACE sample, however, included mainly helpseeking individuals, who may not be typical of the population of people at risk of psychosis. Furthermore, individuals with high levels of cannabis use may be less motivated to seek treatment than our subjects. In the Edinburgh High Risk study, those who used cannabis or other illicit drugs were more likely to have psychotic-like symptoms at baseline (Miller et al, 2001), although no longitudinal data examining substance use as a risk factor for transition to psychosis in this cohort is available. Given the contrasting results to date, further research is required within prodromal and ultra high risk samples to clarify the role of cannabis as a risk factor for the onset of psychosis.

\section{Stress}

Stressful life events may precede onset of psychotic illnesses (Bebbington et al, 1993) and psychotic relapses in established disorders (Hirsch et al, 1996); this has led to the hypothesis that adverse life experiences may actually precipitate onset of psychotic episodes in vulnerable individuals. Furthermore, it has been suggested that minor life events or day-to-day hassles may cause more stress than major, although infrequent, events such as deaths or separations (Malla \& Norman, 1992). It is also likely that it is the subjective experiences of stress, rather than stressful events per se, that may be relevant to outcome. For example, Myin-Germeys et al (2001) found that individuals with psychotic illnesses reacted with more intense negative affect to subjective appraisals of stress in their daily life than healthy controls, suggesting a difference in tolerance to stress between the groups. Thus the experience of stressful or distressing events and the inability to adequately cope with them may affect the worsening of psychotic symptoms and the development of psychotic disorder. Among prodromal and ultra-high risk cohorts, an association at baseline between psychoticlike symptoms and lifetime experience of major stressors has been reported (Miller et al, 2001), and a significant association was found between 'impaired tolerance of normal stress' and psychosis onset in the
PACE cohort (Yung et al, 2005). A longitudinal study in the PACE sample examining subjectively assessed stress to objective measures including cortisol levels is discussed below.

\section{Neurocognitive}

Cognitive deficits are recognised as one of the core features of schizophrenia and have been associated with functional outcome (Green, 1996). Recent research has attempted to detect the presence of cognitive deficits prior to illness onset, as they may represent neurocognitive trait markers for schizophrenia. One promising marker is working memory, which is consistently impaired throughout the course of the illness (Goldman-Rakic, 1994). We recently demonstrated that working memory is impaired prior to the onset of psychotic illness in a group of young people at ultra high risk for psychosis (Brewer et al, 2005). This research has also identified immediate verbal recall deficits prior to illness onset, where rapid registration and efficient recall may be the cognitive processes that indicate compromised prefrontal functioning. Olfactory identification deficits have also been found to occur prior to psychosis onset, and have been shown to be worse in patients later diagnosed with schizophrenia (Brewer et al, 2001). However, despite this, the predictive value of neurocognitive variables has proved to be disappointingly poor.

\section{Neurobiological}

Hypothalamic-pituitary-adrenal (HPA)-axis dysfunction may play a role in the development of psychotic disorders. This is supported by the finding of higher cortisol levels (plasma, salivary or urinary) and abnormal circadian cortisol rhythms in patients with psychotic disorders compared to healthy control subjects (Kaneko et al, 1992).

We recently investigated HPA-axis functioning in 12 ultra-high risk participants at the PACE clinic using the combined dexamethasone corticotrophin releasing hormone (DEX/CRH) test (Thompson et al, 2007). Over a 2-year period, 3 of the 12 participants developed a psychosis. Analysis indicated that contrary to expectations participants who did not make the transition to psychosis had on average higher cortisol levels, as well as a greater severity of depression and anxiety symptoms, than participants who subsequently developed psychosis. These preliminary results suggest that dysregu- lated HPA-axis functioning in individuals at ultra high risk for psychosis may be associated more with comorbid depression symptoms than factors specifically related to the process of emerging psychosis illness.

The pituitary gland contains corticotrophs, the cells that produce and secrete adrenocorticotrophic hormone (ACTH), which in turn activates the secretion of cortisol. A recent study found increased pituitary volumes in patients with first-episode psychosis, while individuals with established schizophrenia of at least 5-years' duration had smaller pituitary volumes than controls (Pariante et al, 2004). In a longitudinal investigation of ultra high risk participants at the PACE Clinic, those who developed psychosis had larger pituitary volumes compared with ultra high risk individuals who did not develop psychosis (Garner et al, 2005). This increased volume is thought to reflect an increase in the size and number of corticotrophs.

Studies of brain structure may also be relevant to the HPA dysfunction model of psychotic disorders. It is hypothesised that abnormal HPA-axis responses to stress might result in hippocampal damage, which may then compromise attention, memory and other cognitive skills and ultimately influence the development of positive psychotic symptoms. Reduced hippocampal volume (Velakoulis et al, 1999), has been reported in association with psychotic illnesses.

Hippocampal volumes of PACE ultrahigh risk patients at intake lie midway between those of normal controls and patients with chronic schizophrenia or first-episode psychosis (Phillips et al, 2002b). However, reduced hippocampal volumes in the ultra-high risk cohort at baseline have not been shown to be associated with a heightened risk of later developing psychosis. In fact, ultra-high risk individuals who developed psychosis had larger hippocampi at baseline than those who did not develop psychosis within a 12-month period (Phillips et al, 2002b). This was due to the ultra-high risk individuals who did not develop psychosis (the false-positives) having smaller than average hippocampal volumes. It must be remembered that these false positives are not analogous to normal healthy controls. They are help-seeking and symptomatic with a range of symptoms and psychiatric syndromes. The reduced hippocampal volume in this subsample of patients may therefore reflect this nonpsychotic psychopathology. 
In a subsample of ultra-high risk patients who developed psychosis, we obtained magnetic resonance imaging (MRI) brain scans at baseline (i.e. prior to onset of frank psychosis) and 1 year later (post-psychosis). Scans showed a significant bilateral reduction in grey matter volume in the cingulate region as well as in the left para-hippocampal gyrus, left fusiform gyrus, left orbitofrontal cortex and one region of the left cerebellar cortex (Pantelis et al, 2003). These findings were not present in a group of ultra high risk patients scanned at baseline and follow-up but who did not develop psychosis. Progressive hippocampal volume loss across the early course of illness is also strongly suggested by a much larger cross-sectional study recently reported by our group (Velakoulis et al, 2006). These findings suggest that brain changes occur during the period of transition to and early course of psychosis, and, while the basis of this remains uncertain, it opens up the possibility that stress, dysregulated neurodevelopment and apoptosis may be involved, and that with sufficiently early and effective treatment such changes could be minimised or prevented.

\section{Intervention}

Three published studies suggest that intervention may be able to delay or even prevent onset of psychosis in the ultra-high risk or prodromal group. The first randomised controlled trial was conducted at the PACE clinic from 1996 to 1999 (McGorry et al, 2002). This study compared the effect of intensive cognitive-behaviourally oriented psychotherapy plus low-dose neuroleptic (risperidone) with supportive therapy alone on the development of psychotic disorder. There was a significantly higher rate of transition to psychosis in the control (supportive therapy) group compared to the intervention group at the end of the 6month treatment phase, although this difference was no longer significant at 12month follow-up. This result is thought to indicate a delay in the onset of psychosis in the intervention group. Both groups experienced a reduction in global psychopathology and functioning over the treatment phase. Because psychological treatment and medication were combined in this trial it was not possible to determine which was the active intervention, or whether they were synergistic with each other. A trial of cognitive therapy versus monitoring only conducted by the Early Detection and Intervention (EDIE) group in Manchester showed a significant effect of the treatment (Morrison et al, 2004). A double masked randomised controlled trial of olanzapine versus supportive therapy and monitoring showed a trend for the intervention in effectively preventing or delaying psychosis (McGlashan et al, 2006). Additionally, the prodromal (ultra high risk) patients who received olanzapine reported lower levels of 'prodromal' symptomatology compared to the UHR patients who received placebo. The results of this study highlighted the fine line between benefit and risk in this early stage, since sideeffects were of more concern in this study than in the PACE trial, yet the clinical benefit, at least in terms of transition rates, was more equivocal. In all, results of these first treatment trials suggest that both antipsychotic medication and psychological interventions might have a role in treating the difficulties and problems young people at ultra-high risk experience as well as delaying or preventing the onset of psychosis. There is scope for the investigation of a wide range of other approaches, including neuroprotective agents in the treatment of the ultra-high risk population. The sequence of treatment strategies is also clearly in need of further research and the risk/benefit ratio, made explicit as the key consideration in the staging model, should be the guiding principle. In the meantime, for such patients, clear explanation of the best available evidence (incomplete though it may be, as elsewhere in psychiatry), truly informed consent and patient and family involvement in decision-making is crucial. A conservative approach in relation to antipsychotic use is strongly suggested, with psychosocial and other benign treatment interventions advised as first-line (McGorry et al, 2005). Treatment should be offered in a non-stigmatised primary care, youthfriendly or office setting and engagement and therapeutic alliance are key initial objectives to secure. Careful follow-up and reassurance is vital. Although this area of research has been controversial in some settings, provided these guidelines are followed, we have seen no evidence of harm to patients and much benefit in our youthfriendly service setting over a 12-year period. Ethical issues have been discussed in detail elsewhere (McGorry et al, 2001).

\section{CONCLUSIONS}

Different methods of identifying those at imminent risk of developing a psychotic disorder have been described. These involve using different combinations of genetic, age range and symptomatic risk factors. However, even using these multiple-gate screening models, the majority of those selected still do not develop the disorder. Identifying features within these cohorts which seem to be associated with increased risk is a research priority. This aids our detection of individuals in whom indicated preventive intervention may be justified.

To date several risk factors for progression to more established illness within the ultra high risk (clinical stage $1 b$ ) group have been identified. Schizotypal and positive psychotic features need further investigation to clarify which particular symptoms seem to be most predictive. Other clinical variables that may be of importance in mediating onset include distress, stress, depression, poor functioning and substance use. The ongoing investigation of endophenotypes (see Table 1 ) is also needed, including neurocognitive, neurobiological and physiological.

Undoubtedly, discussion will occur about how high a positive predictive value (PPV) is needed to justify specific treatment, including antipsychotic medication, in the ultra high treatment cohort. Arriving at a PPV of $100 \%$ would be a clear indication for pharmacological treatment in the identified group. However, these individuals who had this 'risk factor' with a PPV of $100 \%$ for psychotic disorder could actually be conceptualised as having the disorder, albeit in its very early stages. That is, they would be stage 2 rather than stage $1 b$ (see Table 1), and treatment would not be indicated prevention, so much as early treatment of diagnosed illness. Until then, however, the field remains in need of ongoing research efforts.

\section{Limitations}

To date sample sizes in the ultra high risk (prodromal) field of research are small. Findings are inconsistent, particularly in relation to cannabis use and neuroanatomical changes. Another limitation is the onset of psychotic disorder is arbitrarily defined and does not differ qualitatively from subthreshold psychosis.

\section{APPENDIX}

Commonly described prodromal features of schizophrenia (modified from Yung, 2006)

Neurotic symptoms

Anxiety 
Restlessness

Anger, irritability

\section{Mood-related symptoms}

Depression

Anhedonia

Guilt

Suicidal ideas

Mood swings

\section{Changes in volition}

Apathy, loss of drive

Boredom, loss of interest

Fatigue, reduced energy

\section{Cognitive changes}

Disturbance of attention and concentration

Preoccupation, daydreaming

Thought blocking

Reduced abstraction

\section{Physical symptoms}

Somatic complaints

Loss of weight

Poor appetite

Sleep disturbance

\section{Attenuated or sub-threshold versions of psychotic symptoms}

Perceptual abnormalities

Suspiciousness

Ideas of reference

Change in sense of self, others

or the world

\section{Other symptoms}

Obsessive-compulsive phenomena

Dissociative phenomena

Increased interpersonal sensitivity

\section{Behavioural changes}

Deterioration in role functioning Social withdrawal

Impulsivity

Odd behaviour

Aggressive, disruptive behaviour

\section{REFERENCES}

Bebbington, P., Wilkins, S., Jones, P., et al (1993) Life events and psychosis. Initial results from the Camberwell Collaborative Psychosis Study. British Journal of Psychiatry, 162, 72-79.

Bell, R. Q. (1992) Multiple-risk cohorts and segmenting risk as solutions to the problem of false positives in risk for the major psychoses. Psychiatry, 55, 370-38I.
Brewer, W. J., Pantelis, C., Anderson, V., et al (200I) Stability of olfactory identification deficits in neurolepticnaive patients with first-episode psychosis. American Journal of Psychiatry, 158, 107-115.

Brewer, W. J., Francey, S. M., Wood, S. J., et al (2005) Memory impairments identified in people at ultra-high risk for psychosis who later develop first-episode psychosis. American Journal of Psychiatry, 162, 71-78.

Broome, M. R., Woolley, J. B., Tabraham, P., et al (2005) What causes the onset of psychosis? Schizophrenia Research, 79, 23-34.

Cornblatt, B., Lencz, T., Correll, C., et al (2002) Treating the prodrome: naturalistic findings from the RAP program. Acta Psychiatrica Scandinavica, 106, 44

Escher, S., Romme, M., Buiks, A., et al (2002) Formation of delusional ideation in adolescents hearing voices: A prospective study. American Journal of Medical Genetics, II4, 913-920.

Fava, G. A. \& Kellner, R. (1993) Staging: a neglected dimension in psychiatric classification. Acta Psychiatrica Scandinavica, 87, 225-230.

Freeman, D. \& Garety, P. A. (2003) Connecting neurosis and psychosis: The direct influence of emotion on delusions and hallucinations. Behavioural Research Therapy, 4I, 923-947.

Garner, B., Pariante, C. M., Wood, S. J., et al (2005) Pituitary volume predicts future transition to psychosis in individuals at ultra-high risk of developing psychosis. Biological Psychiatry, 58, 417-423.

Goldman-Rakic, P. S. (1994) Working memory in schizophrenia. Journal of Neuropsychiatry and Clinical Neurosciences, 6, 348-357.

Green, M. F. (1996) What are the functiona consequences of neurocognitive deficits in schizophrenia? American Journal of Psychiatry, 153 321-330.

Hafner, H., Maurer, K., Loffler, W., et al (1994) The epidemiology of early schizophrenia. Influence of age and gender on onset and early course. British Journal of Psychiatry, 164 (suppl. 23), 29-38.

Hafner, H., Maurer, K., Trendler, G., et al (2005) Schizophrenia and depression: Challenging the paradigm of two separate diseases. A controlled study of schizophrenia, depression and healthy controls. Schizophrenia Research, 77, II-24.

Hanssen, M., Krabbendam, L., de Graaf, R., et al (2005) Role of distress in delusion formation. British Journal of Psychiatry, 187, s55-s58.

Haroun, N., Dunn, L., Haroun, A., et al (2006) Risk and protection in prodromal schizophrenia: ethical implications for clinical practice and future research. Schizophrenia Bulletin, 32, 166-178.

Hirsch, S., Bowen, J., Emani, J., et al (1996) A one year prospective study of the effect of life events and medication in the aetiology of schizophrenic relapse. British Journal of Psychiatry, 168, 49-56.

Johns, L. C., Cannon, M., Singleton, N., et al (2004) Prevalence and correlates of self-reported psychotic symptoms in the British population. British Journal of Psychiatry, 185, 298-305.

Johnstone, E. C., Ebmeier, K. P., Miller, P., et al (2005) Predicting schizophrenia: Findings from the Edinburgh High-Risk Study. British Journal of Psychiatry, 186, 18-25.

Kaneko, M., Yokoyama, F., Hoshino, Y., et al (1992) Hypothalamic-pituitary-adrenal axis function in chronic schizophrenia: association with clinical features. Neuropsychobiology, 25, I-7.

Klosterkotter, J., Schultze-Lutter, F., Gross, G., et a (1997) Early self-experienced neuropsychological deficits and subsequent schizophrenic diseases: an 8-year average follow-up prospective study. Acto Psychiatrica Scandinavica, 95, 396-404.

Klosterkotter, J., Hellmich, M., Steinmeyer, E. M., et al (200I) Diagnosing schizophrenia in the initial prodromal phase. Archives of General Psychiatry, 58, 158-164

Klosterkotter, J., Birchwood, M., Linszen, D., et al (2005) Overview on the recruitment, sample characteristics, and distribution of inclusion criteria of the European Prediction of Psychosis Study (EPOS). European Psychiatry, 20, 48.

Krabbendam, L., Myin-Germeys, I., Hanssen, M., et al (2005) Development of depressed mood predicts onset of psychotic disorder in individuals who report hallucinatory experiences. British Journal of Clinical Psychology, 44, II3-125.

Lencz, T., Smith, C. W., Auther, A., et al (2004) Nonspecific and attenuated negative symptoms in patients at clinical high-risk for schizophrenia. Schizophrenia Research, 68, 37-48.

Linszen, D. H., Dingemans, P. M. \& Lenior, M. E. (1994) Cannabis abuse and the course of recent-onset schizophrenic disorders. Archives of General Psychiatry, 5I, 273-279.

Malla, A. K., \& Norman, R. M. G. (1992) Relationship of life events and daily stressors to symptomatology in schizophrenia. Journal of Nervous and Mental Disease, 180, 664-667.

Mason, O., Startup, M., Halpin, S., et al (2004) State and trait predictors of transition to first episode psychosis among individuals with at risk mental states. Schizophrenia Research, 71, 227-237.

McGlashan, T. H., Zipursky, R. B., Perkins, D., et a (2006) Randomized, double-blind trial of olanzapine versus placebo in patients prodromally symptomatic for psychosis. American Journal of Psychiatry, 163, 790-799.

McGorry, P. D., Yung, A. \& Phillips, L. (200I) Ethics and early intervention in psychosis: keeping up the pace and staying in step. Schizophrenia Research, 5I, 17-29.

McGorry, P. D., Yung, A. R., Phillips, L. J., et al (2002) Randomized controlled trial of interventions designed to reduce the risk of progression to first-episode psychosis in a clinical sample with subthreshold symptoms. Archives of General Psychiatry, 59, 921-928.

McGorry, P. D., Killackey, E., Lambert, M., et al (2005) Australia and New Zealand clinical practice guidelines for the treatment of schizophrenia. Australia and New Zealand Journal of Psychiatry, 39, I-30.

McGorry, P. D., Hickie, I. B., Yung, A. R., et al (2006) Clinical staging of psychiatric disorders: a heuristic framework for choosing earlier, safer and more effective interventions. Australia and New Zealand Journal of Psychiatry, 40, 616-622.

Miller, P., Lawrie, S. M., Hodges, A., et al (200I) Genetic liability, illicit drug use, life stress and psychotic symptoms: Preliminary findings from the Edinburgh study of people at high risk for schizophrenia. Social Psychiatry and Psychiatric Epidemiology, 36, 338-342.

Morrison, A. P., French, P., Walford, L., et al (2004) Cognitive therapy for the prevention of psychosis in people at ultra-high risk: randomised controlled trial. British Journal of Psychiatry, 185, 291-297.

Mrazek, P. J. \& Haggerty, R. J. (eds) (1994) Reducing Risks for Mental Disorders: Frontiers for Preventive Intervention research. National Academy Press.

Myin-Germeys, I., van Os, J., Schwartz, J. E., et a (200I) Emotional reactivity to daily life stress in psychosis. Archives of General Psychiatry, 58, II37-|144. 
Olsen, K. A. \& Rosenbaum, B. (2006) Prospective investigations of the prodromal state of schizophrenia: review of studies. Acta Psychiatrica Scandinavica, II3, 247-272.

Pantelis, C., Velakoulis, D., McGorry, P. D., et al (2003) Neuroanatomical abnormalities before and after onset of psychosis: a cross-sectional and longitudinal MRI comparison. Lancet, 36I, 28I-288.

Pariante, C. M., Vassilopoulou, K., Velakoulis, D., et a (2004) Pituitary volume in psychosis. British Journal of Psychiatry, 185, 5-10.

Phillips, L. J., Curry, C., Yung, A. R., et al (2002a) Cannabis use is not associated with the development of psychosis in an 'ultra' high-risk group. Australia and New Zealand Journal of Psychiatry, 36, 800-806

Phillips, L. J., Velakoulis, D., Pantelis, C., et al (2002b) Non-reduction in hippocampal volume is associated with risk for psychosis. Schizophrenia Research, $\mathbf{5 8}$ 145-158.

Thompson, K., Berger, G., Phillips, L., et al (2007)

HPA axis functioning associated with transition to psychosis: combined DEX/CRH test. Journal of Psychiatric Research, 4I, 446-450.

Thornicroft, G. (1990) Cannabis and psychosis. Is there epidemiological evidence for an association? British Journal of Psychiatry, I57, 25-33.

Tien, A.Y. (1991) Distributions of hallucinations in the population. Social Psychiatry and Psychiatric Epidemiology, 26, 287-292.

van Os, J., Hanssen, M., Bijl, R. V., et al (200I)

Prevalence of psychotic disorder and community level of

ALISON R. YUNG, MD, MPM, FRANZCP, PATRICK D. McGORRY, MD, PhD, FRANZCP, ORYGEN Research

Centre and the Department of Psychiatry, University of Melbourne, Parkville, Victoria, Australia

Correspondence: Alison Yung, ORYGEN Research Centre, Locked Bag 10, Parkville, Victoria, Australia. Tel: +6I 39342 2000; fax +6I 39342 2948; email: aryung@unimelb.edu.au

psychotic symptoms: An urban-rural comparison Archives of General Psychiatry, 58, 663-668.

Velakoulis, D., Pantelis, C., McGorry, P. D., et al (1999) Hippocampal volume in first-episode psychoses and chronic schizophrenia: a high resolution magnetic resonance imaging study. Archives of General Psychiatry. 56, $133-14 \mid$.

Velakoulis, D., Wood, S. J., Wong, M.T. H., et al (2006) Hippocampal and amygdala volumes according to psychosis stage and diagnosis: a magnetic resonance imaging study of chronic schizophrenia, first-episode psychosis, and ultra-high-risk individuals. Archives of General Psychiatry, 63, 139-149.

Yung, A. R. (2006) Identification of the population. In Working with People at High Risk for Developing Psychosis: A Treatment Handbook (eds A. P. Morrison, J. Addington \& S. M. Francey), pp. 7-23. John Wiley.

Yung, A. R. \& McGorry, P. D. (1996) The prodroma phase of first-episode psychosis: past and current conceptualizations. Schizophrenia Bulletin, 22, 353-370.

Yung, A. R., McGorry, P. D., McFarlane, C. A., et al (1996) Monitoring and care of young people at incipient risk of psychosis. Schizophrenia Bulletin, 22, 283-303.
Yung, A. R., Phillips, L. J., Yuen, H. P., et al (2003)

Psychosis prediction: 12-month follow up of a high-risk ('prodromal') group. Schizophrenia Research, 60, 21-32.

Yung, A. R., Phillips, L. J., Yuen, H. P., et al (2004) Risk factors for psychosis in an ultra high-risk group: Psychopathology and clinical features. Schizophrenia Research, 67, |3|-|42.

Yung, A. R., Yuen, H. P., McGorry, P. D., et al (2005) Mapping the onset of psychosis - the Comprehensive Assessment of At Risk Mental States (CAARMS). Australia and New Zealand Journal of Psychiatry, 39 964-971.

Yung, A. R., Buckby, J. A., Cotton, S. M., et al (2006)

Psychotic-like experiences in non-psychotic helpseekers: associations with distress, depression and disability. Schizophrenia Bulletin, 32, 352-359.

Yung, A. R., Stanford, C., Cosgrave, E., et al (2006) Testing the ultra high risk (prodromal) criteria for the prediction of psychosis in a clinical sample of young people. Schizophrenia Research, 84, 57-66.

Zammit, S. (2002) Self reported cannabis use as a risk factor for schizophrenia in Swedish conscripts of 1969 : historical cohort study. British Medical Journal, 325 1199-1204. 